\title{
Study and Analysis of AGC and PFC to Power Grid Frequency Control
}

\author{
Jun $\mathrm{Li}^{1, \mathrm{a}}$, Dapeng Liao ${ }^{2, \mathrm{~b}}$, Huicong $\mathrm{Li}^{2, \mathrm{c}}$, Hui Zhang ${ }^{2, \mathrm{~d}}$ and Ting Wang ${ }^{2, \mathrm{e}}$ \\ ${ }^{1}$ State Grid Shandong Electric Power Research Institute, Jinan 250002, China \\ ${ }^{2}$ Dispatching \& Control Center, Shandong Electric Power Corporation, Jinan 250001, China \\ alijun_sdu@hotmail.com, b38799335@qq.com, 'Ihc_111@163.com,

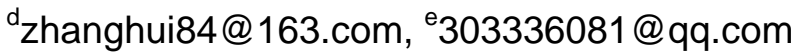

\begin{abstract}
Keywords: grid frequency; automatic power generation control; primary frequency compensation; control strategy
\end{abstract}

\begin{abstract}
The interconnections of regional power network, wind power and other new energy connected to power grid make the operation of power grid more difficultly. The stability of power grid frequency mainly depends on primary frequency compensation (PFC) and automatic power generation control (AGC) of thermal power units. By analyzing the characteristics of them, the actual strategy response to frequency of accidents, and the mechanism of coordination of them were studied. The proposed control strategy can effectively control units to achieve the stability of power grid frequency.
\end{abstract}

\section{Introduction}

Power grid frequency is one of important indicators of power quality, it reflects the balance between the actual electricity usage and generation of active power, and it is an important control parameter of the power system operation. The frequency stability of the electric network is one of the most primary conditions of power system normal running. As a crucial basic indicator, mains frequency demands a balance between generating and power utilization. Currently, frequency compensation is mainly used for thermal power units.

As Chinese power industry's rapid development, the regional power grid interconnection has become a reality. As a carrier for achieving most optimum allocation of electric power resources, interconnected power network is the important base for region and province power networks to fully realize net-function. As is well known, power grid frequency is one of the three indicators of power quality, it reflects the balance between the generation of active power and load, and it is an important control parameter of the power system operation. The safety and efficiency of the majority of users of electrical equipment and power equipment have a close relationship with power grid frequency. Equipment of users is generally driven by the motor, the same as equipment of power plant. The frequency fluctuation has a critical influence over them. With the development of science and technology, some new electronic equipment and precision processing equipment put forward higher request to power grid frequency, the frequency fluctuations will lead to lower product quality or equipment damage. According to the IEEE 446-1995 "Recommended practice for emergency and standby power systems for industrial and commercial applications" and BS EN50160:1995 "Voltage characteristics of electricity supplied by public distribution systems", to many devices, the maximum tolerance of the frequency fluctuations is $\pm 0.5 \mathrm{~Hz}$.

The occurrence of unexpected failures in the power grid will break the balance between the generation of active power and load. With the development of the power system, single device failures bring a growing loss of generating power grid. Only relying on manually adjust the power generation output will require a longer time to achieve a new balance. Solve these problems. The way is only one that is the techniques of PFC and AGC. It's a good idea to use different logical controls according to its own regulation performance, to maintain the balance of power system supply and demand automatically, thus ensuring the quality of the power system frequency. 


\section{Analysis of PFC and AGC}

Frequency regulation of power system is inherent in the use of system load frequency characteristics, as well as the role of the generator's governor to prevent the system frequency deviation from the standard adjustment method. The grid frequency is determined by the value of generated energy and electricity consumption. If the value of generated energy is equal to the electricity consumption, the grid frequency is stably. If the value of generated energy is greater than the electricity consumption, the grid frequency will increase. If the value of generated energy is less than the electricity consumption, the grid frequency will reduce.

Characteristic of PFC. The primary frequency regulation, refers to the grid frequency deviation from the rating, control system of unit in the grid automatically control the increase or decrease of the unit active power, to limit the changes of grid frequency, so that the grid frequency maintain the stability of automatic control process. The static frequency modulation characteristic of electric network is the foundation of frequency stability of the electric network that determined by frequency modulation. The schematic of PFC is shown in Fig.1.

$$
\Delta P_{L 0}=B 0+A B=\left(K_{G}+K_{L}\right) \Delta f=K_{S} * \Delta f
$$

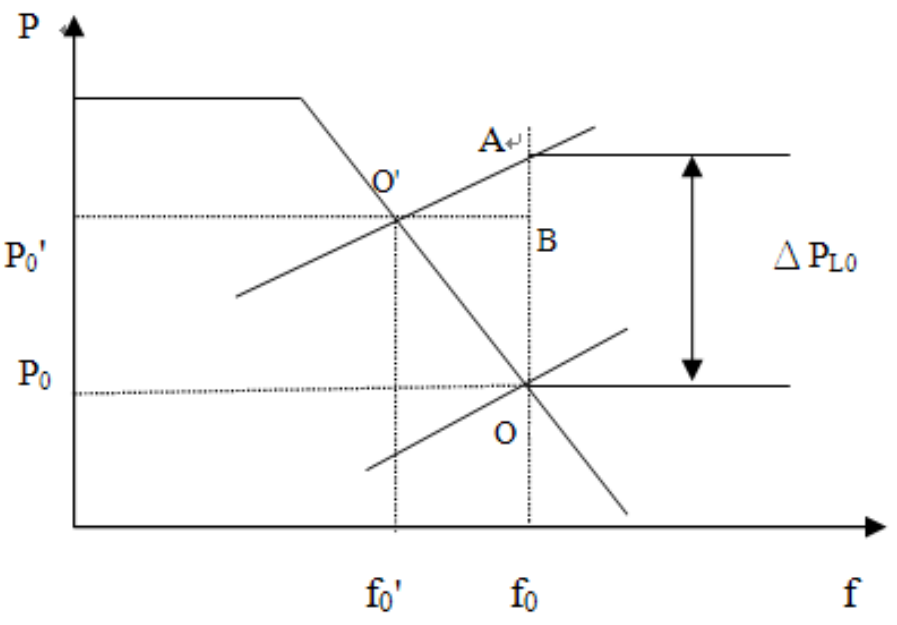

Fig.1. Schematic of PFC

Ks are the unit power regulation of system, depending on the generator unit power regulation. When the increases or decreases of system load, Ks mark the amount that the system frequency increases or decrease at the joint action of generators and loads.

According to the statistics of IEEE, the characteristic time constants of PFC in power system is about 10 seconds, it is fast response to the changes of system frequency. Thermal power unit primary frequency regulation effect of time is short. Different types of thermal power unit, because of the different heat storage capacity. The action time of PFC is 0.5 to 2 minutes inequality. PFC is adjusted by the adjusting methods of the differ characteristic method, its advantage is the adjustment of all units only with a system frequency related, the interaction of units is small.

According to the characteristics of PFC, it's known that the function of PFC control is, to automatic balance the first load component, namely those fast, amplitude smaller, random load, and to buffer the abnormal situation of load mutation.

Characteristic of AGC. AGC is one of the important measures to regulate the frequency and active power of power grid and ensure its security and economic operation. PFC is a kind of important way of the frequency control system, but, because of its attenuation characteristic and differ regulation, can't to control the system frequency depending solely on it. To realize zero error output adjustment of frequency, it must rely on the frequency of the AGC. The schematic of PFC is shown in Fig.2.

$$
\Delta P_{L 0}=\Delta P_{G 0}+B C+A B=\Delta P_{G 0}+K_{S} * \Delta f
$$

If $\Delta \mathrm{P}_{\mathrm{L} 0}=\Delta \mathrm{P}_{\mathrm{G} 0}$, namely generators increase $\Delta \mathrm{P}_{\mathrm{L} 0}$ load power as assumed, then $\Delta \mathrm{f}=0$, that is, to achieve a so-called no droop. No droop in dashed lines in Fig.2. 


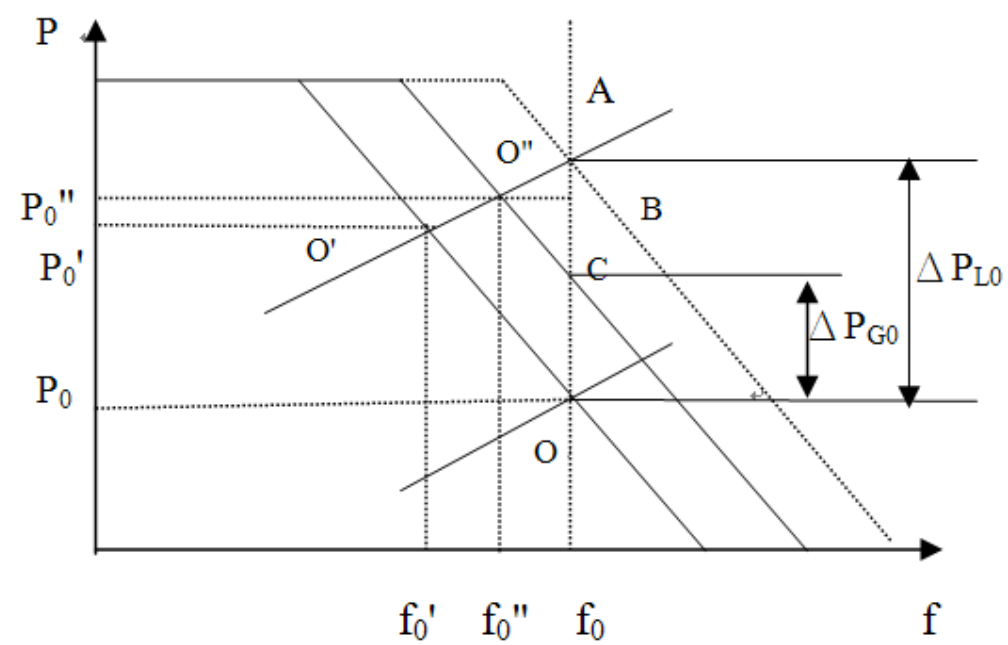

Fig.2. Schematic of AGC

The adjustment way of SFC for system frequency is no deviation. In the CCS of the thermal power unit, because of the energy conversion process time limit, it's much slower that SFC on system load change response than PFC, and its response time general need 1-2 minutes. Based on the characteristics of the PFC, it is known that due to the time of secondary regulation response is relatively slow, therefore cannot adjust those fast load random fluctuation, but it can effectively adjust the minutes level and longer cycle load fluctuation.

\section{Control Strategy}

Generally speaking, the PFC in domestic most power plants adopts the power circuit correction (CCS) and speed governing side feed-forward (DEH) control strategy. DEH is implementation stage, difference, open loop control, ensure frequency compensation's rapidity, CCS is correction stage, no difference, closed loop control, ensure the accuracy and persistent of frequency compensation. In order to guarantee the quality of regulation, speed and power signal of PFC have been done by selecting mid-valve of three same signals, to be shared with DEH and CCS system, to ensure the consistency of judgment on both sides of the signal.

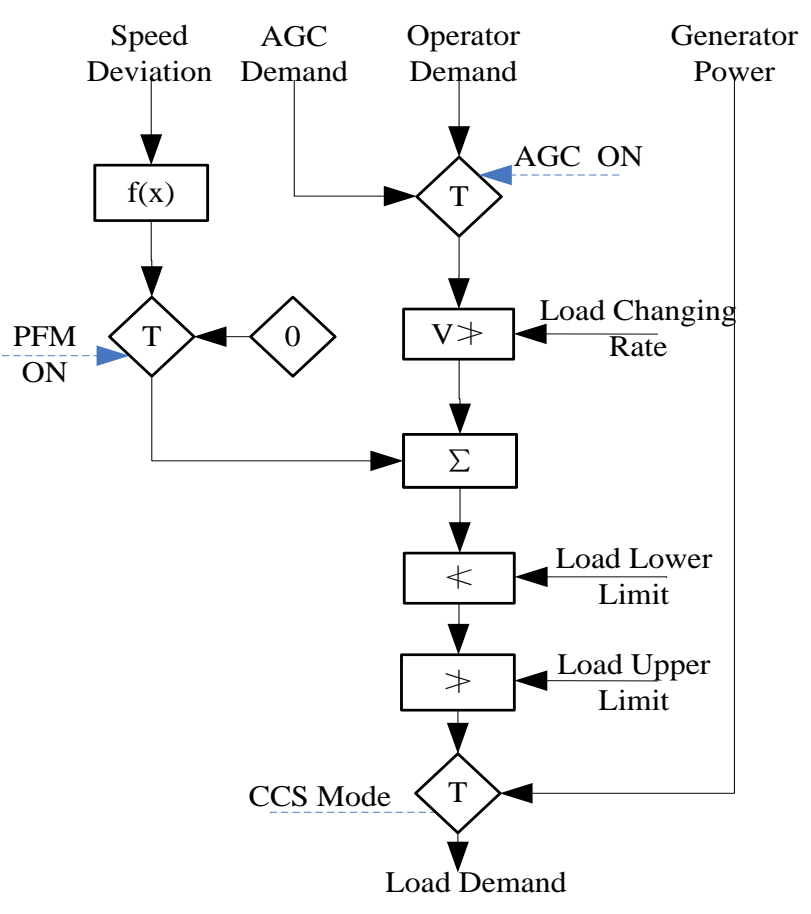

Fig.3. Logic of PFC in CCS side

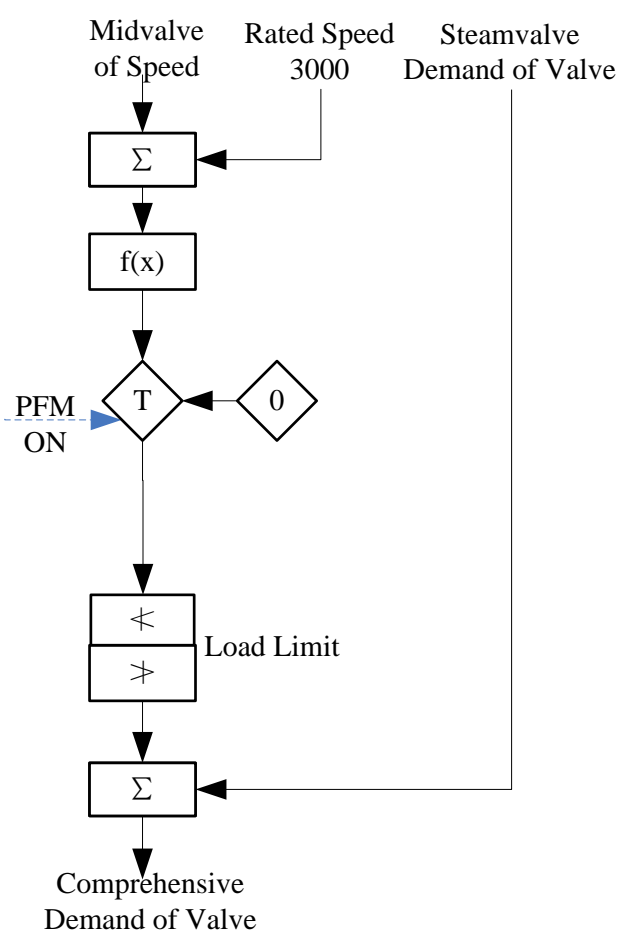

Fig.4. Logic of PFC in DEH side 
As shown in Fig.3, speed deviation signal is converted to the corresponding frequency modulation power through the function generator $\mathrm{f}(\mathrm{x})$, and added to the unit load after rate limiting, and after load high and low limit, forms the final actual load demand, ensure frequency compensation demand quickly and correctly and accurately.

The control logic of PFC in DEH side is shown in Fig.4, speed deviation of rated speed and mid-valve of actual speed represents the frequency deviation. After the operation of $f(x)$ that have dead zone, power increment of the unit frequency modulation can be achieved. Through the frequency modulation load amplitude limit, the valve position demand of frequency modulation is generated, to be added directly in steam flow demand of valve, it is to be delivered to the valve of turbine satisfy the quickness of frequency modulation.

\section{Practical Frequency Regulation}

According to the Q/GDW 669-2011 “Guide of primary frequency compensation test for thermal power generating units", steady-state speed regulation is $5 \%$. When the rated power $\left(\mathrm{P}_{0}\right)$ is less than 250MW, the max frequency compensation load is $10 \% \mathrm{P}_{0}$. When $\mathrm{P}_{0}$ is more than $250 \mathrm{MW}$ and less than $350 \mathrm{MW}$, the max frequency compensation load is $8 \% \mathrm{P}_{0}$. When $\mathrm{P}_{0}$ is more than $350 \mathrm{MW}$ and less than $500 \mathrm{MW}$, the max frequency compensation load is $7 \% \mathrm{P}_{0}$. When $\mathrm{P}_{0}$ is more than $500 \mathrm{MW}$, the max frequency compensation load is $6 \% \mathrm{P}_{0}$.

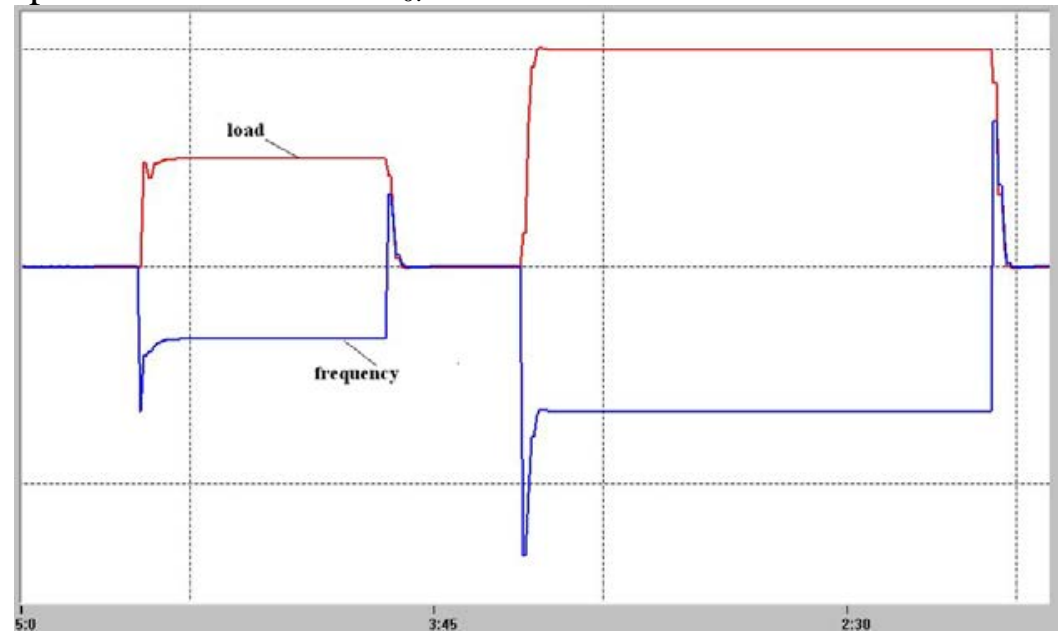

Fig.5. The frequency dynamic process of simulation

Assume that only one unit in a power grid, when power grid frequency reduced from $50 \mathrm{~Hz}$ to $49.8 \mathrm{~Hz}$, after unit's PFC, frequency stabilizes at $49.9 \mathrm{~Hz}$ finally. When power grid frequency reduced from $50 \mathrm{~Hz}$ to $49.6 \mathrm{~Hz}$, after unit's PFC, frequency stabilizes at $49.8 \mathrm{~Hz}$ finally. The frequency dynamic process is shown in Fig.5. The reason is that PFC is droop control, the grid frequency cannot return to $50 \mathrm{~Hz}$. Depend on the relationship between primary frequency modulation speed difference and primary frequency modulation loads, the frequency only increase half of the frequency deviation.

One power grid accident, the grid frequency drop rapidly, grid frequency after 12 seconds reaches the lowest, it is $49.563 \mathrm{~Hz}$. After 30 seconds, dynamic ACE began to action and absorbs the deficiency power, then, in the provinces of emergency call the spinning reserve, AGC action under the joint action, the system frequency is gradually increased, in 221 seconds system restore to $49.80 \mathrm{~Hz}$, after 334 seconds back to $49.95 \mathrm{~Hz}$. The frequency dynamic process is shown in Fig.6. In the first 30 seconds, the grid frequency modulation mainly depends on PFC. In fact, the lowest is $49.563 \mathrm{~Hz}$, after PFC, grid frequency is $49.76 \mathrm{~Hz}$. According to the analysis, PFC mostly restores the frequency to $49.79 \mathrm{~Hz}$. There is only $0.03 \mathrm{~Hz}$ deviation.

So, in order to better quality of frequency modulation, need to AGC combined with PFC. When big power grid frequency fluctuation occur, AGC should be according to the size of the ACE, dynamic adjustment the number of units into emergency mode, as soon as possible to return to $50 \mathrm{~Hz}$. That is, when there is a large power grid frequency deviation, ACE load range at the emergency regulation area, to be based on the magnitude of the load is missing the appropriate amount of 
operating units, in order to quickly make up the power shortfall.

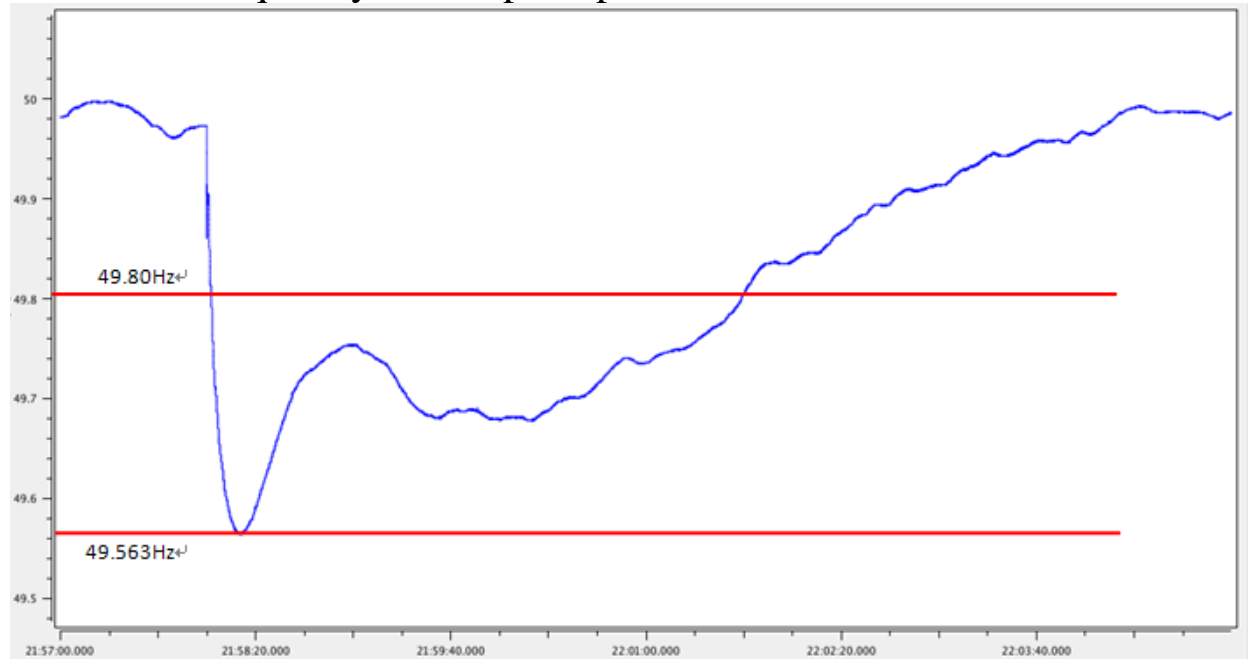

Fig.6. The frequency dynamic process of one power grid accident

\section{Conclusions}

As two important technical means of frequency control of the power system, PFC and AGC are of great significance to the stability of the frequency. By analyzing thermodynamic system of thermal power unit, primary frequency compensation control strategy response to frequency of accidents and the relationship between PFC and AGC, points out that they should work together to meet the requirements of the grid frequency adjustment. It is not conducive to adjust the grid frequency. Meanwhile, in order to maintain the stabilization of frequency, the regulation capability of each control area is evaluated and restricted by control performance standard. The update and field tests of generator PFC and AGC function should be carried out.

\section{References}

[1] D.R.Coyghanowr, Process Systems Analysis and Control, McGraw-Hill, New York, 1991.

[2] YIN Feng. Test and research on CCS-joined primary frequency regulation of thermal power units [J]. Electric Power, 2005, 38(3), pp.74-77.

[3] YU Da-ren, GUO Yu-feng, XU Ji-yu. The primary frequency regulation stability of parallel turbo generators [J]. Proceedings of the CSEE, 2000, 20(9):59-63.

[4] Harris, T.J. Assessment of closed loop performance [J]. Canadian Journal of Chemical Engineering, 1989, 67:856-861.

[5] WANG Zhen-yi, XIE Yi-gong, YIN Cheng-quan, et al. Research on the coordination of AGC and primary frequency regulation based on CPS [J]. Power System Protection and Control, 2007, 37(19):22-25.

[6] CHEN Liang, CHEN Hui-kun. Analysis on primary frequency regulation of generator units in Guangdong Power System [J]. Guangdong Electric Power, 2007, 21(8): 8-12.

[7] ZHU Wei, TAN Xi-yi, TANG Ying-jie, et al. Analysis and research on primary frequency modulation of the turbine generation unit [J]. Automation of Electric Power Systems, 2008, 32(24): 52-55.

[8] Zheng Tao, Gao Fuying. On-line monitoring and computing of unit PFR characteristic parameter based on PMU [J]. Automation of Electric Power Systems, 2009, 33(11):57-61, 71.

[9] Liu Nian, Xie Chi. Teng Fusheng. Study of safety and stability problems for power system [J].Sichuan Electric Power Technology, 2004(1): 1-6 